\title{
MPPT Based Slope Compensated Current Mode Controlled Charging of a Battery in Standalone PV-Battery System \\ ${ }^{* 1}$ Ankit Kant Saxena, ${ }^{2}$ Binod Shaw, ${ }^{3}$ Shashikant \\ 1,2,3 National Institute of Technology, Raipur - 492010, India \\ Email: ankitkantsaxena@gmail.com,binodshaw2000@gmail.com,shashikantkaushaley20@gmail.com
}

Received: 06th October 2019, Accepted: 31st January 2020, Published: 29th February 2020

\begin{abstract}
In order to improve the life span of the battery in a PV (photovoltaic) system, controlled charging is necessary. To do the task battery management system is put in place which monitors the charging and discharging of the batteries. Maximum power point technique is quite popular for extraction of the maximum power from the solar panel so charging of the battery with the maximum current extracted from the PV panel at variable irradiance is very effective for fast charging of the battery. In this paper P\&O based MPPT current mode controlled charging is proposed. The unheralded technique of slope compensation is used which is critical in solving the problem associate with current mode design. This method is easy to use and implemented and produce superior results. Constant current charging is compared with the proposed technique and the results are depicted. The PV system is implemented in MATLAB Simulink and the required performance is obtained.
\end{abstract}

Keywords

Renewable Energy, Battery Charging, MPPT, Solar Energy, Battery Management System

\section{Introduction}

The conventional energy crisis and the negative effects of the use of fossil fuel to the environment forces us to think for the alternative source of energy which can be available in abundance and doesn't aid in the degradation of the environment. The renewable energy sources are the future of power generation. Solar power is the most popular and widely used energy. In rural and distant places grid connection is still not available. Standalone PVBattery system is the simple solution for electrification and hence control strategy should be design accordingly for efficient use. [1] PV power is intermittent in nature; battery becomes the most easily available \& effective combination for PV. For effective and reliable operation, battery charging in a controlled manner is necessary. So that battery can power the load in night-time efficiently. Battery is quite costly and if proper conditioning is not done it can get damaged. Controlled charging and discharging is essential to increase the life-span of the battery. Since the efficiency of solar panel is quite low, we need to extract the maximum available power from it. Different MPPT (Maximum power point tracking) techniques are available in the literature. [2]

A battery can be charged differently depends upon the type and the system available, it can be classified as a) Constant current (CC) b) constant voltage (CV) c) Constant Current-Constant voltage (CC-CV) d) Pulse charging e) MPPT based charging. [3] [4]MPPT charging is preferred when there is a need to get a good balance between controlled and fast charging and still extract maximum power from the PV source. In MPPT charging, the battery charging current will follow the insolation changes in the PV panel so that the battery Charging current is different in accordance with the different time (insolation) during the day. [5] [6]

Some of the advantages of MPPT charging is 1) efficient use of solar energy 2) fast charging of the battery 3) easy for maintaining the good and quick SOC (state of charge) response of the battery. The disadvantage of this technique is if the insolation keeps on changing drastically in accordance the charging current will change so that because of sudden high current battery may get damaged.

In this paper MPPT based charging is analyzed and implemented in MATLAB SIMULINK and the results are provided. In addition, in this paper constant current charging is also analyzed and compared with the MPPT based charging.

\section{Components of the PV-Charging Circuit: \\ System Design}

The system design of PV based battery charger is given in the Fig 1. The system comprises of PV module which is then coupled with modified buck converter. Different battery types have different specifications and limitations. MPPT algorithm is applied which is based on P\&O technique in this system. Finally controlling structure is provided which takes input as the inductor current, current from the PV terminals and the battery voltage, to decide and control the pulses given to the gate terminals of the IGBT (insulated gate bipolar transistor) and switches ON-OFF according to the signal given as output from the controlling structure. 


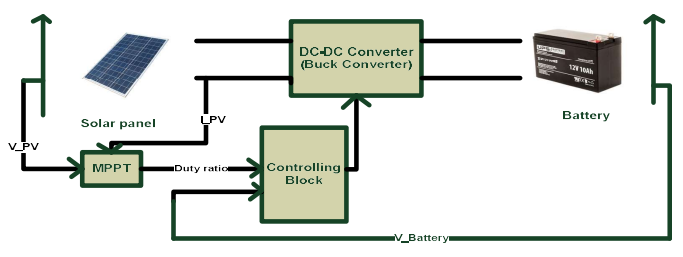

P\&O MPPT Technique

Fig. 1: System Design

This MPPT algorithm checks the $d p / d v$ regularly to determine the updated duty ratio. [7] According to which we design the coding pattern. The logic are as follows

$\frac{d p}{d v}= \begin{cases}>0 & V<V m \\ =0 & V=V m \\ <0 & V<V m\end{cases}$

The flowchart of the $\mathrm{P} \& \mathrm{O}$ method is given below:

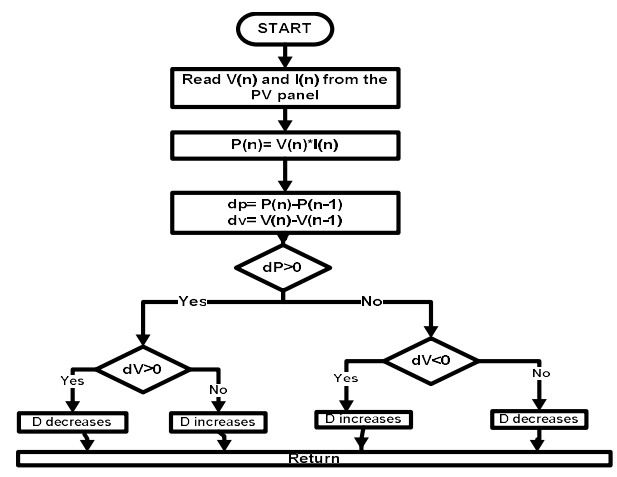

DC-DC Converter

Fig. 2: Flowchart of P\&O MPPT Algorithm

In this model we use the buck converter whose structure is given below in Fig 3. The additional component we added in the circuit is the battery which is put in parallel with the capacitor and the load which is resistive. We call this circuit Modified buck circuit. The rest of the working is the same as explained in literature. [8] [9]

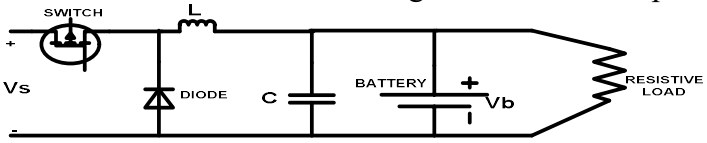

\section{Control Technique}

Fig. 3: DC-DC Converter Configuration

Fig 4 represents the structure inside of the controlling block which tracks the changes in the environmental conditions and change the charging current accordingly. As I_inductor < (I_pv-I_slope), comparator output will be low so reset will be 0 and for every rising pulse of Clock signal flip-flop output will be 1 which is then compared with the output of the Duty cycle coming from the MPPT and according it is given to the switch as the gate pulse. 


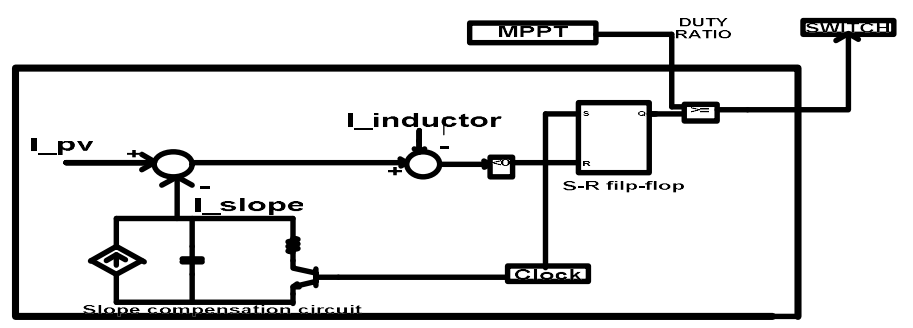

Fig. 4: Controlling Block

The main component inside the controlling block is the Slope compensation circuit which is used to solve the critical problem of the current mode controlled which is divergence of the inductor current when the duty ratio is greater than 0.5 . Fig 5(b) demonstrates the critical problem in which cycle after cycle the disturbance starts growing, so it never converges to steady state value of inductor current.
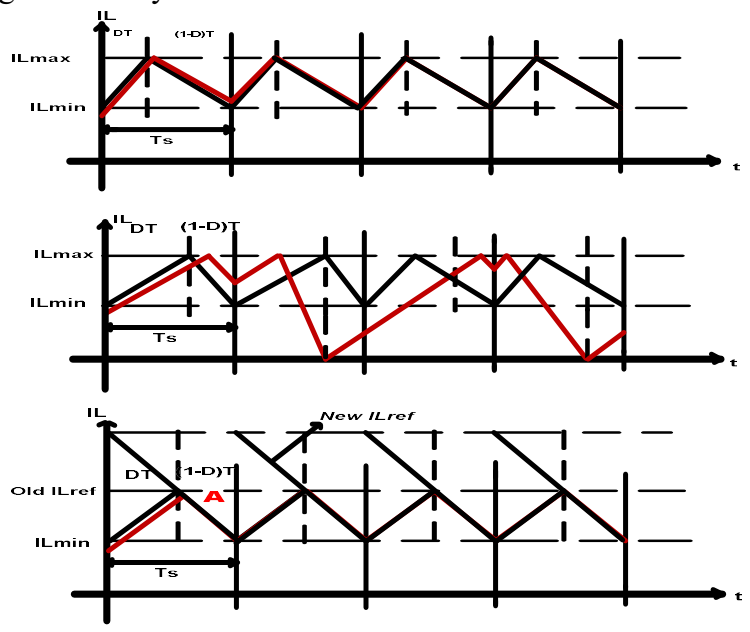

Fig. 5: Waveforms Associated with Inductor Current a) when $D<0.5$ b) when $D>0.5$ c) Corrected for $\mathrm{D}>0.5$

To solve the above specified problem here slope compensated circuit is placed in which we draw a slope line with slope similar to the falling slope or discharging state slope, which is continuous in every Ts. A saw tooth like waveform will be formed and it will become the new IL_ref which is shown in Fig 5(c), at the instant A, the flip-flop will be reset and error will be reduced in one quick cycle and then it follows the steady state value.

The slope of the new $i L_{-}$ref = same as the falling slope of $i L$.

This method is called slope compensation and the circuit is called as Slope compensation circuit as shown in Fig 6 [10] Here there is a CCCS (current controlled current source) which sense the battery voltage continuously and changes the magnitude of the CCCS. Since it is an integrator it keeps on integrating and doesn't come to 0. Hence we keep a switch which is supplied from CLK. Whenever the CLK signal comes the capacitor discharges through the switch, we also put a small resistance in switch to limit discharging current.

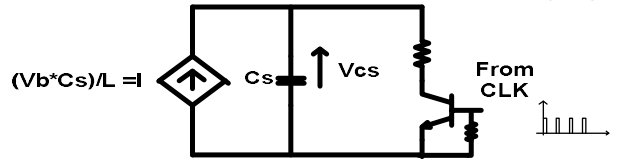

Fig. 6: Slope Compensated Circuit

When we applied MPPT algorithm in the circuit, Iref keeps changing depending on upon the insolation so ILref can be set to draw maximum power from the source and it charge up the battery and load as the best power available from the source.

\section{Results and Discussion}

Simulation Results

The simulation is performed in MATLAB-SIMULINK and the following results were produced. Fig 7 shows the battery characteristics. Here we use Lithium-ion battery in simulation but this technique holds true to different types of batteries. 


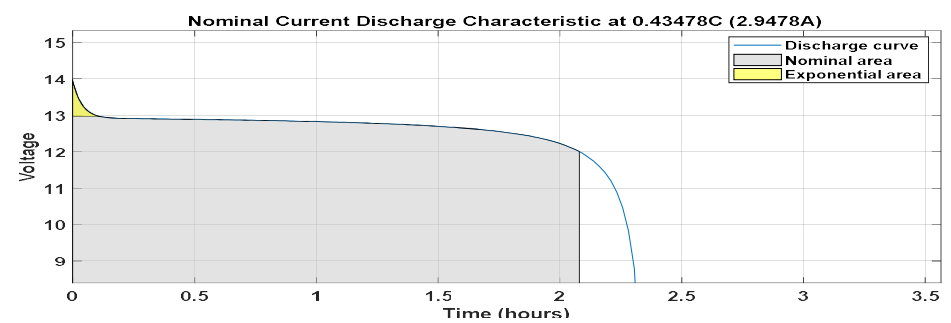

Fig. 7: Battery Characteristics

The variable irradiance in Fig 8a shows the which is taken in simulation. Fig $8 \mathrm{~b}$ shows the SOC (state of charge) of the battery which shows that the battery SOC percentage is increasing so it clearly means the battery is charging and the slope of the SOC curve depends on the magnitude of current coming into the battery. As we can see at $0.2 \mathrm{sec}$ the irradiance increases so the charging current increases and consequently slope of the SOC curve changes and becomes more steep. Fig8c shows the Battery Voltage which we can see changes very slightly when the irradiance is changed. The battery current is shown in Fig 8d, as the current's magnitude is negative, we say that the battery is charging and the battery receives maximum amount of current at noon as the irradiance is maximum then, so the battery charges quickly.

Fig 9a depicts comparison between the result of the aforementioned technique and the standard constant current charging. Fig $9 \mathrm{~b}$ shows that the state of charge of the same battery when charged with different technique. The technique proposed in this paper charges the battery faster than the standard method of Constant current.
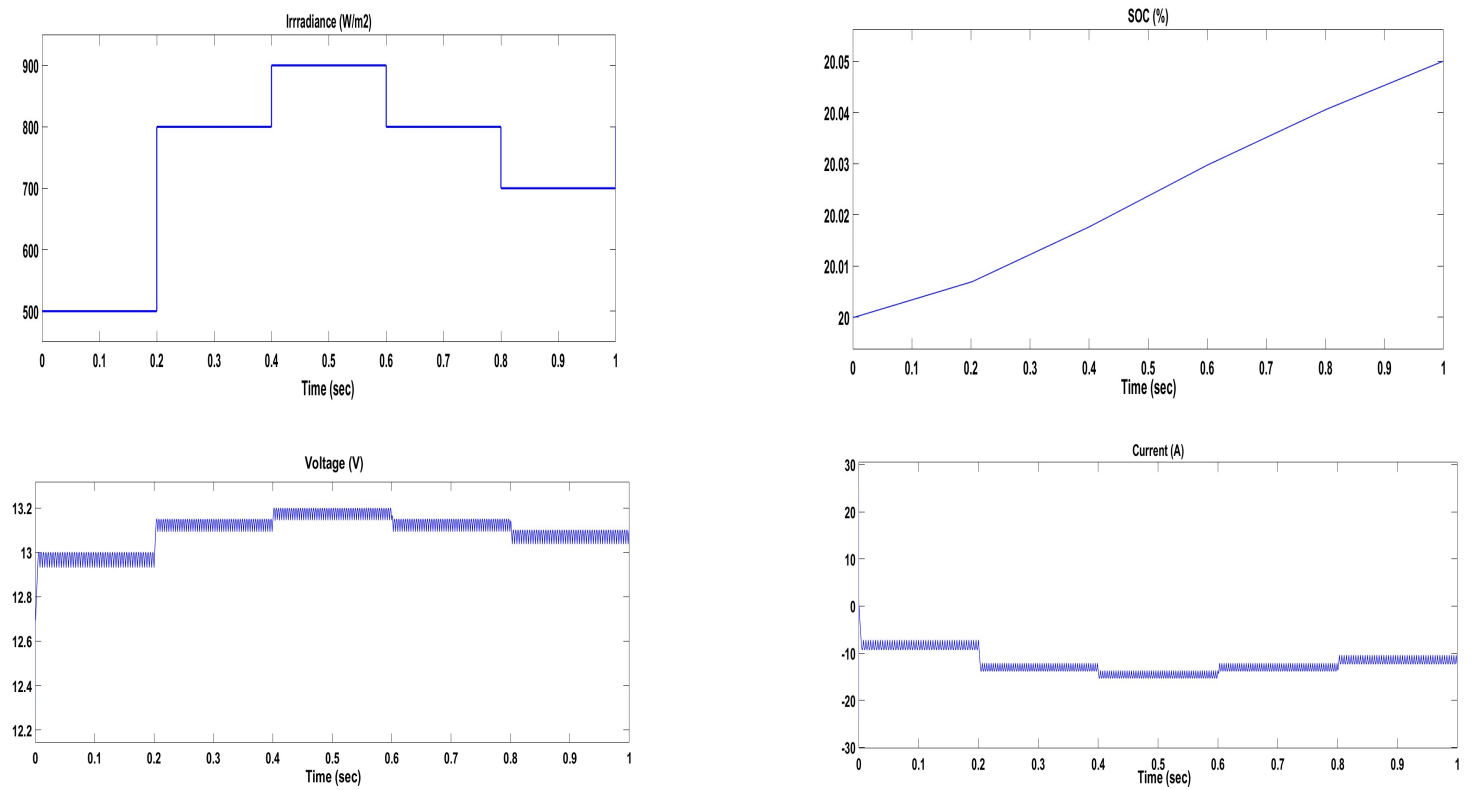

Fig. 8 a): Variable Irradiance b): State of Charge of the Battery c): Battery Voltage d): Battery Current
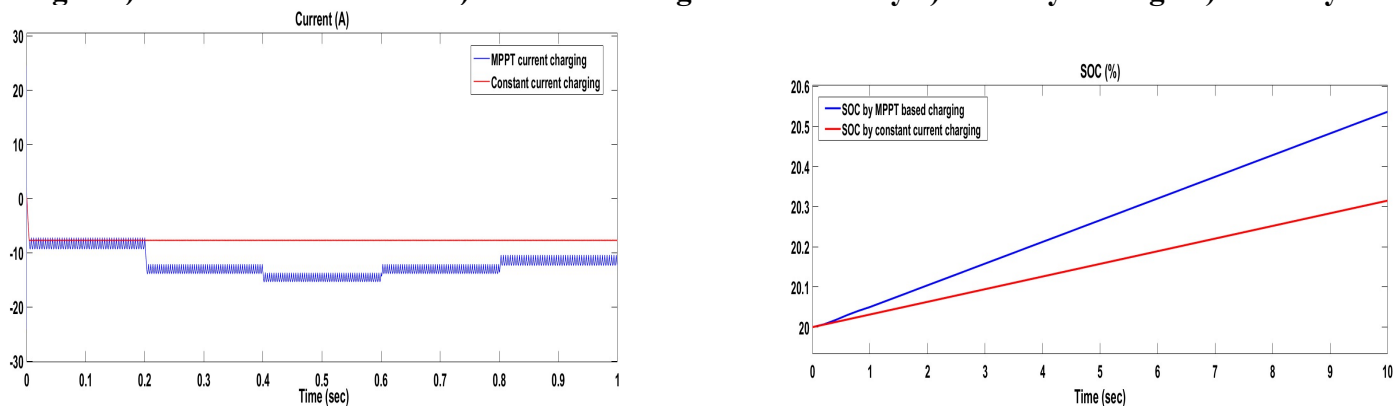

Fig. 9 a): MPPT based Charging Current VS Constant Current Charging b): SOC of MPPT Charging vs SOC of Standard Constant Current Charging 


\section{Conclusion}

In this paper, comprehensive and detailed design of the MPPT algorithm and the controlling structure of PV system is provided which can be applied easily and used for controlled charging of the battery in PV-Battery household applications. Also the results of standard constant current charging are compared with the MPPT charging and the advantages of the technique is provided. All the results in simulation verifies the theoretical calculation aspect.

\section{References}

1. Liao, Z. and Ruan, X. (2009) 'A novel power management control strategy for stand-alone photovoltaic power system', 2009 IEEE 6th International Power Electronics and Motion Control Conference, IPEMC '09. IEEE, 3, pp. 445-449. doi: 10.1109/IPEMC.2009.5157429.

2. Rezk, H. and Eltamaly, A. M. (2015) 'A comprehensive comparison of different MPPT techniques for photovoltaic systems', Solar Energy. Elsevier Ltd, 112, pp. 1-11. doi: 10.1016/j.solener.2014.11.010.

3. Cope, R. C. and Podrazhansky, Y. (2008) 'The art of battery charging', pp. 233-235. doi: 10.1109/bcaa.1999.795996.

4. Bhattacharjee, A. (2012) 'Design and Comparative Study of Three Photovoltaic Battery Charge Control Algorithms in MATLAB/SIMULINK Environment', International Journal of Advanced Computer Research, 2(5), pp. 129-137.

5. Padhee, S., Pati, U. C. and Mahapatra, K. (2016) 'Design of photovoltaic MPPT based charger for lead-acid batteries', 2016 IEEE International Conference on Emerging Technologies and Innovative Business Practices for the Transformation of Societies, EmergiTech 2016. IEEE, pp. 351-356. doi: 10.1109/EmergiTech.2016.7737365.

6. Lixin, P. et al. (2008) 'Analysis of photovoltaic charging system based on MPPT', Proceedings - 2008 Pacific-Asia Workshop on Computational Intelligence and Industrial Application, PACIIA 2008, 2, pp. 498501. doi: 10.1109/PACIIA.2008.357.

7. Selmi, T. et al. (2014) 'P\&O MPPT implementation using MATLAB/Simulink', 2014 9th International Conference on Ecological Vehicles and Renewable Energies, EVER 2014. IEEE, pp. 1-4. doi: 10.1109/EVER.2014.6844065.

8. Hauke, B. (2011) 'Basic Calculation of a Buck Converter's Power Stage', Application Report, (December 2011), pp. 1-8.

9. Issaadi, W. (2016) 'An improved MPPT converter using current compensation method for PV-applications', International Journal of Renewable Energy Research, 6(3), pp. 894-913.

10. Zhong, J. and Liu, S. (2011) 'Design of slope compensation circuit in peak-current controlled mode converters', 2011 International Conference on Electric Information and Control Engineering, ICEICE 2011 Proceedings. IEEE, pp. 1310-1313. doi: 10.1109/ICEICE.2011.5778040. 